\title{
Galaxy coordinates
}

\section{Accurate positions for 17124 galaxies including 3301 new companions of UGC galaxies}

\author{
G. Paturel ${ }^{1}$, C. Petit ${ }^{1}$, R. Garnier ${ }^{1}$, and P. Prugniel ${ }^{1}$ \\ CRAL-Observatoire de Lyon, F-69561 Saint-Genis Laval Cedex, France
}

Received February 14; accepted April 11, 2000

\begin{abstract}
This paper gives accurate coordinates and diameters for 3301 galaxies, companions of UGC galaxies. (about 2764 companions were not yet available in electronic form). In addition previously poor equatorial coordinates are re-measured for 13823 galaxies. These coordinates which have an accuracy of $5^{\prime \prime}$ or better will be used for facilitating the cross-identification with forthcoming catalogues of millions of galaxies.
\end{abstract}

Key words: galaxies - catalog

\section{Introduction}

This series of papers aims mainly at improving the accuracy of equatorial galaxy coordinates in order to facilitate the cross-identification with the very large catalogue we built from the Digitized Sky Survey using an automatic galaxy recognition program (Paturel et al. 2000). Nevertheless, it is also important to enter new galaxies well identified visually, especially when they are either close to large galaxies or when they have a low surface brightness. Indeed, these two characteristics make difficult an automatic recognition.

This paper presents two independent sections, both aiming at improvement of equatorial coordinates of galaxies. In Sect. 2 we describe the catalogue of companions of UGC galaxies. Most of these galaxies are not yet entered in large databases. Then, in Sect. 3 we give a short description of additional 13935 coordinate measurements. These measurements are performed in a way similar to the one described in our previous paper (Paturel et al. 1999; Paper II).

\footnotetext{
Send offprint requests to: G. Paturel

* Full Tables 1 and 2 are available in electronic form at CDS via anonymous ftp to cdsarc.u-strasbg.fr (130.79.128.5) or via http://cdsweb.u-strasbg.fr/Abstract.html
}

\section{Catalogue of UGC galaxy companions}

Many years ago Peter Nilson (1973) produced his famous UGC catalogue with nearly 13000 galaxies larger than one arcminute. This catalogue was integrated a long time ago in general extragalactic databases like NED or LEDA. At the end of the UGC catalogue Nilson gave many additional pieces of information about the UGC galaxies and their environment. For instance, small galaxies surrounding a UGC galaxy and listed in the CGCG catalogue (Zwicky et al. 1961-1968) are given with their original CGCG coordinates. Further, Nilson identified many small companions around his UGC galaxies.

There is no clear definition of such companions. They are simply "close" to a UGC galaxy. Nilson did not measure the equatorial coordinates for these galaxies but instead made an identification in polar coordinates with respect to the central UGC galaxy. This relative position is given by the position angle $\beta$ (measured in degrees from north through east) and the angular separation $\rho$ (measured in arcminute from the center of the parent UGC galaxy). In addition, Nilson gives major and minor axes (in arcminute) of these small companions.

This catalogue of UGC companions was keypunched by one of us (CP) ten years ago. This was a difficult task because of the very compact printing format of this catalogue. If the coordinates of these companion galaxies had been deduced at that time from the polar coordinates, any deplacement of the parent galaxy would have completely distorted the look of the field. Of course this could have been solved by moving the companions at the same time but it is not obvious to know which galaxy is a companion and which is not. Our catalogue remained ten years on our computer disk.

Today, thanks to the remarkable work by Cotton et al. (1999), all UGC galaxies have accurate equatorial coordinates. This suggested to us that we use this catalogue as a source of equatorial coordinates for the central UGC galaxies. However, instead of calculating the equatorial coordinates from the polar ones we prefered to use the plate 


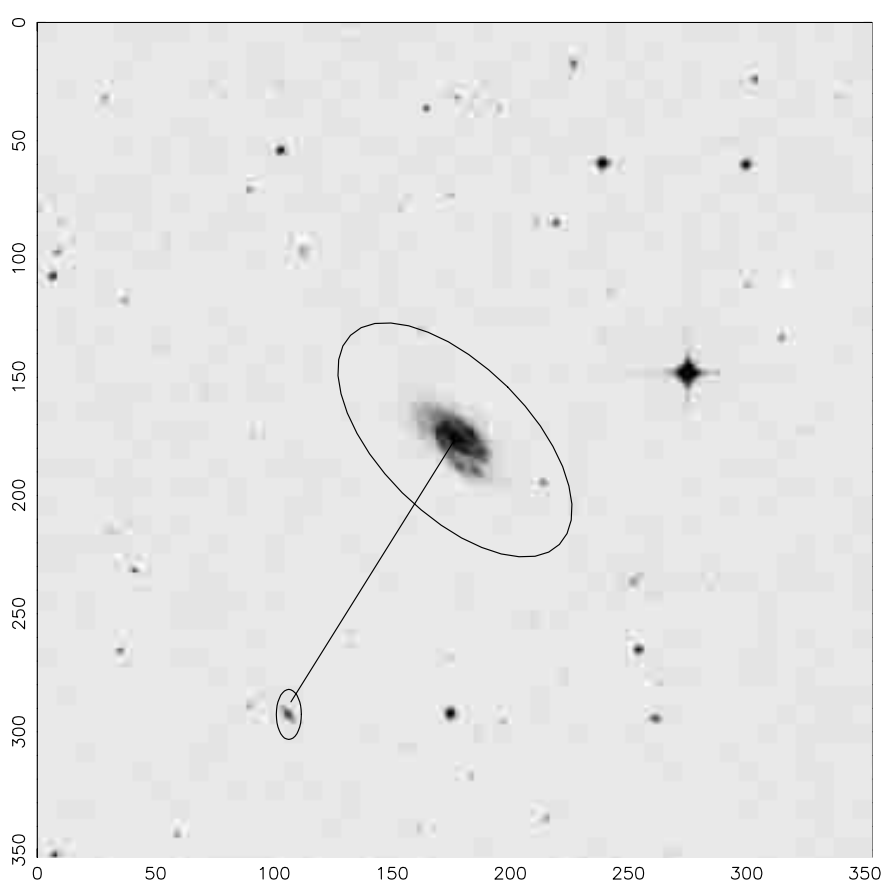

Fig. 1. A typical image from the Digitized Sky Survey got from ESO. The frame is $10^{\prime} \times 10^{\prime}$. North is on the upper side, East is on the left side. The large galaxy in the center is the parent UGC galaxy (UGC 5). The companion is the small galaxy at the end of the line. The diameter of the companion is drawn according to twice the Nilson's measurements

solution given by the Digitized Sky Survey. The first reason is that the accuracy of the measurements of position angle and angular separation is not good enough to provide us with accurate equatorial coordinates. The position angle is known with an accuracy of one or two degrees and the angular separation is known with an accuracy of $6^{\prime \prime}$ to $10^{\prime \prime}$ leading to an uncertainty of $14^{\prime \prime}$ at a distance of $5^{\prime}$ from the center of the parent galaxy. The second reason is that the uncertainties on both axes (R.A. and DEC.) would have been correlated in a complicated manner.

Instead, the polar coordinates are used for an easy identification of companion galaxies by displaying simultaneously the DSS image, centered on the parent UGC galaxy, and the location of companions. In Fig. 1 we present such a display.

The coordinates are obtained by clicking on the center of the companion. This is done with special routines developed from the PGPLOT package. The center is estimated visually.

\subsection{Treatment of images}

We selected first UGC galaxies with companions and made the histogram of the radial vectors of their companions. This histogram is given in Fig. 2.

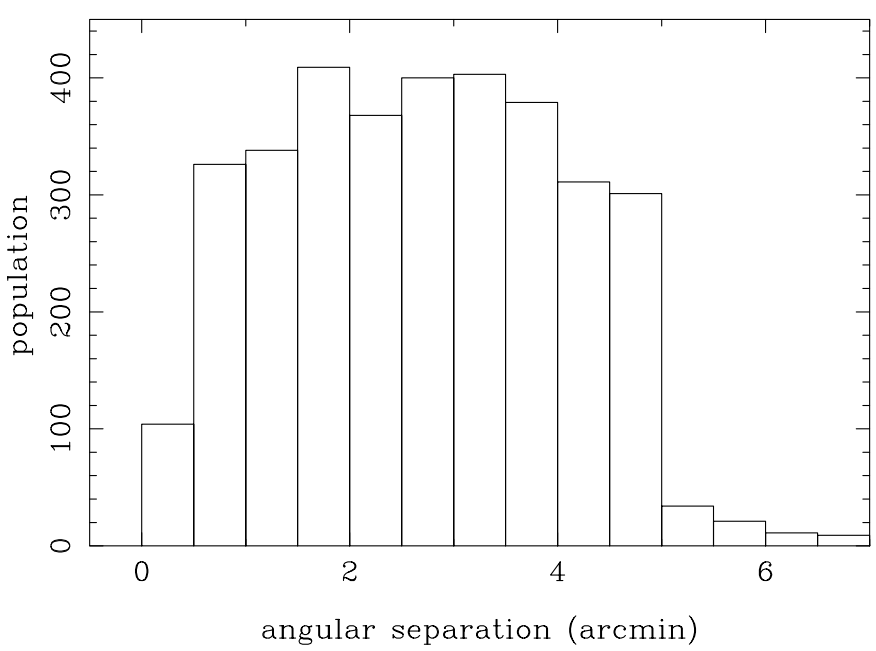

Fig. 2. Histogram of angular separations of parent galaxy and companions. We considered only companions with a separation smaller or equal to $5^{\prime}$

The coordinates of the UGC galaxies are taken from the LEDA database ${ }^{1}$. For efficiencies sake we loaded all DSS images (i.e. about 2600 images) from the ESO facility.

Then, the measurements were done. Sometimes, the identification is not possible either because we have a choice between two companions or because of an error in the input catalogue. These cases were treated individually by refering to the original UGC catalogue or by analysing the field carefully.

This work results in 3301 new accurate coordinates for companions of 2597 UGC galaxies. Most of these companions are not available in large databases. For each companion we give the major axis and minor axis diameters as measured by Nilson.

\subsection{The final catalogue}

The catalogue is now loaded in LEDA but it will be distributed in an electronic form via the Astronomy and Astrophysics archives. Here we simply reproduce a sample page (Table 1). This table gives measurements for 3301 galaxies (539 were already known).

The following data are given:

Column 1: PGC number according to LEDA database (Paturel et al. 1989).

Column 2: Name according to some well-known catalogues, if applicable. In Table 3 we give the acronyms used for these catalogues.

Column 3: Right Ascension and Declination for equinox 2000 (in hours, minutes, seconds and tenths and degrees, arcminutes and arcseconds).

Column 4: Major axis diameter in arcminute measured

\footnotetext{
1 After having loaded the astrometry of UGC galaxies by Cotton et al. (1999).
} 
Table 1. Catalogue of UGC galaxy companions. The full list is availbale in electronic form

\begin{tabular}{lllrrrr}
\hline PGC & Name & $\begin{array}{l}\text { R.A.2000 DEC2000 } \\
\text { h min sec deg }\end{array}$ & $\begin{array}{r}D \\
\text {, }\end{array}$ & d & ugc-parent \\
\hline 0000037 & MCG 4- 1- 9 & $000031.6+261819$ & 0.25 & 0.15 & \\
0215025 & & $000047.8+285602$ & 0.30 & 0.10 & 12901 \\
0000089 & MCG 2- 1- 9 & $000113.3+130836$ & 0.80 & 0.30 & 12906 \\
0215026 & & $000114.1+344059$ & 0.20 & 0.10 & 12904 \\
0000092 & MCG 2- 1- 10 & $000115.1+130647$ & 0.50 & 0.15 & 12906 \\
0215027 & & $000129.5+130551$ & 0.50 & 0.40 & 12906 \\
1422918 & & $000145.3+130601$ & 0.40 & 0.40 & 12906 \\
0215028 & & $000211.2+173337$ & 0.50 & 0.30 & 12916 \\
0215029 & & $000212.8+125643$ & 0.40 & 0.40 & 12919 \\
0215030 & & $000216.3+125501$ & 0.30 & 0.20 & 12919 \\
0212450 & & $000227.4+445333$ & 0.35 & 0.25 & 00002 \\
0000185 & IC 5379 & $000240.7+163602$ & 0.70 & 0.35 & 00001 \\
0212451 & & $000253.6+185041$ & 0.50 & 0.30 & 00003 \\
0212453 & & $000308.0+155836$ & 0.40 & 0.20 & 00007 \\
0212452 & & $000313.6-015809$ & 0.30 & 0.15 & 00005 \\
0212454 & & $000318.0+083641$ & 0.60 & 0.20 & 00010 \\
0000234 & KUG 0000+271 & $000325.0+272412$ & 0.30 & 0.30 & 00013 \\
0212457 & & $000329.1+041747$ & 0.30 & 0.20 & 00015 \\
0212455 & & $000334.4+220456$ & 0.50 & 0.35 & 00011 \\
0000252 & CGCG 408- 17 & $000337.7+083810$ & 0.70 & 0.30 & 00010 \\
$\ldots$ & & & & & \\
\hline
\end{tabular}

by Nilson (1973).

Column 5: Minor axis diameter in arcminute measured by Nilson.

Column 6: UGC Name of the parent galaxy.

It is to be noted that two galaxies are companion of two different UGC galaxies. (PGC 12267 is companion of UGC 2639 and UGC 2651; PGC 212567 is companion of UGC 408 and UGC 423. In the catalogue they are given on the same line).

\section{Additional list of new accurate equatorial coordinates}

At the beginning of this work the percentage of galaxies with good coordinates in LEDA was about $90 \%$. We selected galaxies still having poor coordinates (standard deviation less than 10"). About 18500 galaxies were listed for which DSS images were extracted from ESO in Münich. Then, our procedure of measurement was applied leading to 13823 accurate coordinates which are listed in Table 2. The following data are given:

Column 1: PGC number according to LEDA (Paturel et al. 1989).

Column 2: Name according to Table 3, if applicable.

Column 3: Right Ascension and Declination for equinox 2000 (in hours, minutes, seconds and tenths and degrees, arcminutes and arcseconds).

Column 4: Flag to mention an uncertainty on name.

After having loaded this list and the UGC-companion list $97.8 \%$ of the galaxies (about 190000 galaxies) in LEDA had accurate coordinates ${ }^{2}$. In practice, 365 galaxies presented an inconsistency of names between LEDA and NED. These cases were analyzed with special care. They are noted with an asterisk in Table 2. This shows the urgent need of homogeneization of galaxy designation. Indeed, some galaxies identified in old catalogues with very poor coordinates and very poor description cannot be retrieved in a secure manner in the new catalogues. Then, giving such a name to this or that object is just a matter of convention. We should either abandon the old, inaccurate designation (this is what seems suitable to us) or decide that a given old name designates a given object (the same convention must be adopted by all databases). This requires regular exchanges and dialogues between the different existing general databases.

\section{Accuracy of galaxy coordinates}

According to our previous tests (Paper II) an accuracy of about $5^{\prime \prime}$ to $7^{\prime \prime}$ is obtained for large galaxies (diameter of about $1.7^{\prime}$ ). This uncertainty comes essentially from the uncertainty in the estimate of the galaxy center. For smaller galaxies we expect better accuracy. In order to test the present measurements with small galaxies we measured 285 faint galaxies measured by Munn et al. (1997).

\footnotetext{
${ }^{2}$ We recently loaded our 2.7 million galaxy catalogue extracted from the DSS. The percentage of accurate coordinates in LEDA jumped to $99.9 \%$ but the same 4272 galaxies remain with uncertain coordinates. For these galaxies it is actually an identification problem, not simply a problem of measurement.
} 
Table 2. New measurements of equatorial coordinates. The full table is available in electronic form

\begin{tabular}{lll}
\hline PGC & Name & $\begin{array}{l}\text { R.A.2000 DEC2000 } \\
\text { h min sec deg }{ }^{\prime}{ }^{\prime \prime}\end{array}$ \\
\hline 0089489 & & $000008.4-603304$ \\
0141432 & & $000009.8-633540$ \\
0089490 & & $000018.2-605343$ \\
0000027 & MCG -1- 1- 18 & $000023.3-065612$ \\
0165937 & IRAS 23578-5307 & $000023.8-525031$ \\
0000041 & & $000025.8-054640$ \\
0000036 & IC 5373 & $000029.0+324656$ \\
0000046 & & $000029.7-054636$ \\
0000037 & MCG 4- 1- 9 & $000031.6+261819$ \\
0000040 & MCG 0- 1- 17 & $000035.6-014550$ \\
0097568 & IRAS 23583+1813 & $000054.2+183022$ \\
0141433 & & $000058.8-604956$ \\
0000079 & IC 5374 & $000104.6+043006$ \\
0097569 & IRAS 23586-0116 & $000113.3-005937$ \\
0000122 & MCG -7- 1- 7 & $000130.9-404912$ \\
0141435 & & $000137.7+172919$ \\
0100354 & & $000201.2+145837$ \\
0000152 & MCG -5- 1- 25 & $000205.4-303709$ \\
0000150 & FAIR 1065 & $000205.4-512056$ \\
$\ldots$ & & \\
\hline
\end{tabular}

Table 3. Acronyms used in Tables 1 and 2 for galaxy identification

\begin{tabular}{ll}
\hline Acronym & Reference \\
\hline CGMW & Catalogue of Galaxies Behind the Milky Way; Saito et al., 1990-1991; \\
& Roman et al., 1996-1998 \\
DRCG & Dressler Catalogue of Cluster of Galaxies; Dressler, 1980 \\
ESO & European Southern Observatory catalogue; Lauberts, 1982 \\
FAIR & Fairall lists; Fairall; 1977-1988 \\
HICK & Compact Groups of Galaxies; Hickson, 1993 \\
NGC & New General Catalogue; Dreyer, 1889 \\
IC & Index Catalogue; Dreyer, 1895-1910 \\
CGCG & Catalogue of Galaxies and Cluster of Galaxies; Zwicky et al. 1961-1968 \\
nZW & Catalogue of Selected Compact and Post-eruptive Galaxies; Zwicky, 1971; \\
& Zwicky et al., 1975 ( $n$ is the number of the list from 1 to 8) \\
nSZW & Zwicky Compact Galaxies in two southern fields; Rodgers et al., 1978 \\
& (n is the number of the list from 1 to 2) \\
IRAS & IRAS Point Source Catalogue, 1988 \\
KAZA & Kazaryan lists; Kazaryan, 1979-1983 \\
KCPG & Karachentsev Catalogue of Pair of Galaxies; Karachentsev 1972 \\
KUG & Kiso Survey of Ultraviolet Excess Galaxies: Takase and Miyauchi-Isobe, 1993 \\
MCG & Morphological Catalogue of Galaxies: Vorontsov-Vel'jaminov et al. 1963-1974 \\
MK & Markaryan lists; Markarian 1967-1981 (list 1-15) \\
POX & Catalogue of active galaxies; Kunth; 1981 \\
RB & Rood and Baum Catalogue: Rood and Baum, 1967 \\
UGC & Uppsala General Catalogue; Nilson, 1973 \\
UGCA & Catalogue of Selected non-UGC Galaxies; Nilson, 1974 \\
UM & University of Michigan lists; Mac Alpine et al., 1977-1981 \\
VCC & Virgo Cluster Catalogue; Bingelli et al., 1985 \\
WEIN & Galaxies behind the Milky Way; Weinberger et al., 1980 \\
\hline
\end{tabular}




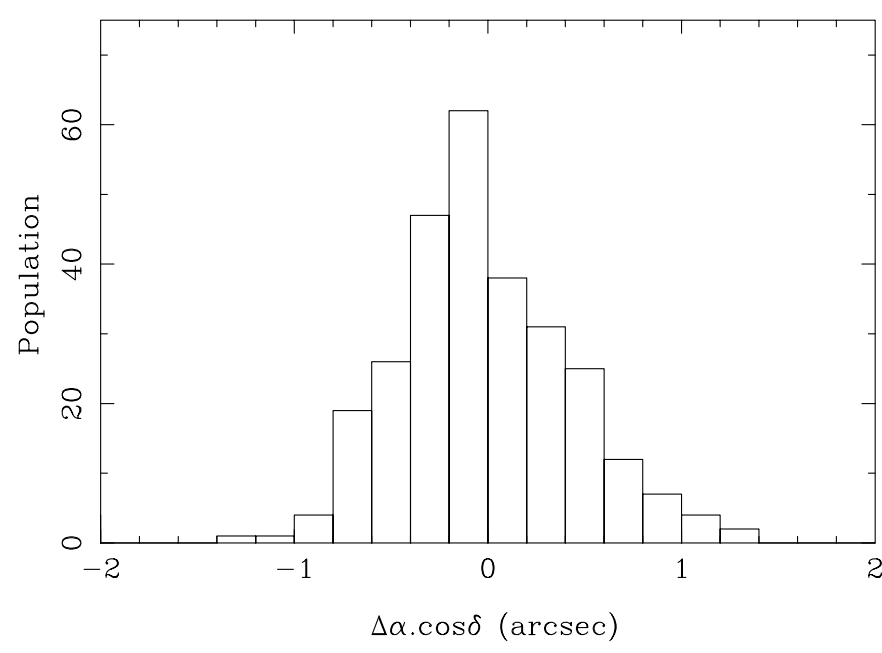

Fig. 3. Histogram of the differences between the Right Ascensions given by Munn et al. and this paper. The standard deviation is $0.6^{\prime \prime}$

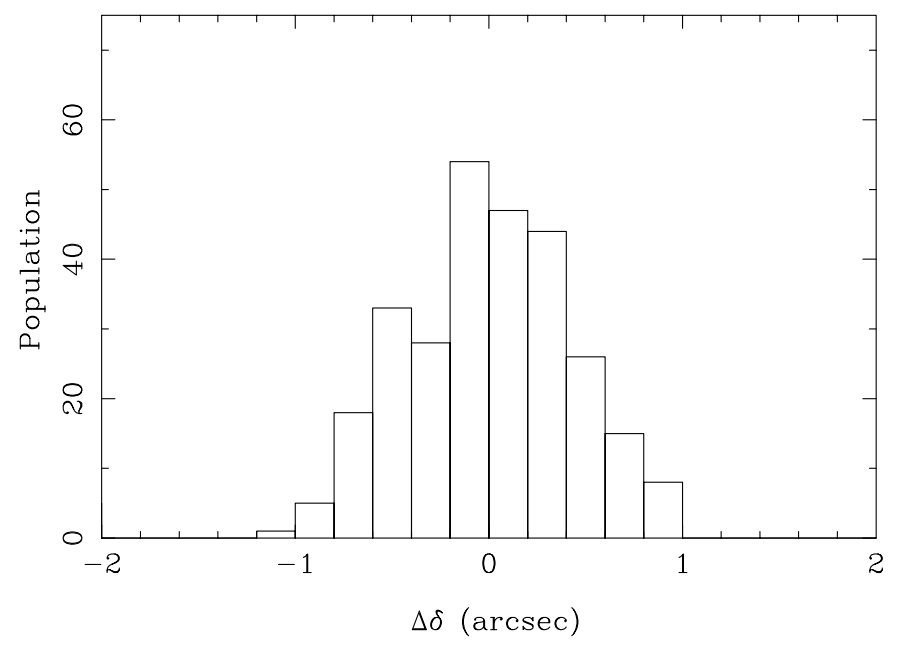

Fig. 4. Histogram of the differences between the Declination given by Munn et al. and this paper. The standard deviation is $0.8^{\prime \prime}$

In Figs. 3 and 4 we present the histograms of the differences between our measurements and those given by Munn et al., for Right Ascensions and Declinations, respectively. There is no significant deviation from zero. For the Right ascension we obtained: $\Delta \alpha \cos \delta=0.02 \pm 0.04^{\prime \prime}(n=$ $285)$. For Declination we obtained: $\Delta \delta=0.08 \pm 0.05^{\prime \prime}$ $(n=285)$.

The standard deviation is $0.6^{\prime \prime}$ and $0.8^{\prime \prime}$ for Right Ascensions and Declinations, respectively. This confirms that the accuracy of coordinates calculated with the DSS plate solution mostly depends on the accuracy of the definition of the galaxy center. For small galaxies (diameter smaller than $1^{\prime}$ ) the accuracy is better than $1^{\prime \prime}$.

Acknowledgements. We thank H.G. Corwin for carefully reading and checking the manuscript of this paper. We made use of the ESO facility in Münich for retrieving images from the Digitized Sky Survey produced at the Space Telescope Science
Institute under US Government grant NAG W-2166. It is based on photographic data obtained using The UK Schmidt Telescope and the Palomar Sky Survey.

The UK Schmidt Telescope was operated by the Royal Observatory Edinburgh, with funding from the UK Science and Engineering Research Council, until 1988 June, and thereafter by the Anglo-Australian Observatory. Original plate material is copyright (c) the Royal Observatory Edinburgh and the Anglo-Australian Observatory. The plates were processed into the present compressed digital form with their permission. We made use of the NASA/IPAC Extragalactic Database (NED) which is operated by the Jet Propulsion Laboratory, Caltech under contract with the National Aeronautics and Space Administration.

\section{References}

Binggeli B., Sandage A., Tammann G.A., 1985, AJ 90, 1681 Cotton W.D., Condon J.J., Arbizzani E., 1999, ApJS 125, 409 Dreyer J.L.E., 1889, MemRAS 49, 1 (NGC)

Dreyer J.L.E., 1895, MemRAS 51, 185 (IC)

Dreyer J.L.E., 1910, MemRAS 59, 105 (IC)

Dressler A., 1980, ApJS 42, 565

Fairall A.P., 1977, MNRAS 180, 391

Fairall A.P., 1979, MNRAS 188, 349

Fairall A.P., 1980, MNRAS 192, 389

Fairall A.P., 1981, MNRAS 196, 417

Fairall A.P., 1983, MNRAS 203, 47

Fairall A.P., 1984, MNRAS 210, 69

Fairall A.P., 1988, MNRAS 233, 691

Hickson P., 1993, Astro. Lett. Comm. 29, 1-207

IRAS Point Source Catalogue, 1988, Version 2. Washington: US Govt. Printing Office (PSC)

Karachentsev I.D., 1972, in Borchkhadze T.M. (ed.), 1974, Astrofizika 10, 493

Kazaryan M.A., 1979, Astrofizika 15, 5

Kazaryan M.A., 1979, Astrofizika 15, 193

Kazaryan M.A., Kazarian E.S., 1980, Astrofizika 16, 17

Kazaryan M.A., Kazarian E.S., 1982, Astrofizika 18, 512

Kazaryan M.A., Kazarian E.S., 1983, Astrofizika 19, 213

Kunth D., Sargent W.L.W., Kowal C., 1981, A\&AS 44, 229

Lauberts A., 1982, The ESO/Uppsala Survey of the ESO(B) Atlas, European Southern Observatory (ESO)

MacAlpine G.M., Smith S.B., Lewis D.W., 1977a, ApJS 34, 95 (List 1)

MacAlpine G.M., Smith S.B., Lewis D.W., 1977b, ApJS 35, 197 (List 2)

MacAlpine G.M., Smith S.B., Lewis D.W., 1977c, ApJS 35, 203 (List 3)

MacAlpine G.M., Lewis D.W., 1977c, ApJS 36, 587 (List 4)

MacAlpine G.M., Williams G.A., 1981, ApJS 45, 113 (List 5)

Markaryan B.E., 1967, Astrofizika 3, 55 (List 1)

Markaryan B.E., 1969a, Astrofizika 5, 443 (List 2)

Markaryan B.E., 1969b, Astrofizika 5, 581 (List 3)

Markaryan B.E., Lipovesky V.A., 1971, Astrofizika 7, 511 (List 4)

Markaryan B.E., Lipovesky V.A., 1972, Astrofizika 8, 155 (List 5)

Markaryan B.E., Lipovesky V.A., 1973, Astrofizika 9, 487 (List 6) 
Markaryan B.E., Lipovesky V.A., 1974, Astrofizika 10, 307 (List 7)

Markaryan B.E., Lipovesky V.A., 1976a, Astrofizika 12, 389 (List 8)

Markaryan B.E., Lipovesky V.A., 1976b, Astrofizika 12, 657 (List 9)

Markaryan B.E., Lipovesky V.A., Stepanyan D.A., 1977a, Astrofizika 13, 225 (List 10)

Markaryan B.E., Lipovesky V.A., Stepanyan D.A., 1977b, Astrofizika 13, 397 (List 11)

Markaryan B.E., Lipovesky V.A, Stepanyan D.A., 1978, Astrofizika 15, 201 (List 12)

Markaryan B.E., Lipovesky V.A., Stepanyan D.A., 1979a, Astrofizika 15, 363 (List 13)

Markaryan B.E., Lipovesky V.A., Stepanyan D.A., 1979b, Astrofizika 15, 549 (List 14)

Markaryan B.E., Lipovesky V.A., Stepanyan D.A., 1981, Astrofizika 17, 619 (List 15)

Munn J.A., Koo D.C., Kron R.G., Majewski S.R., Bershady M.A., Smetanka J.J., 1997, ApJS 109, 45

Nilson P, 1973, Acta Univ. Uppsala, Ser. V1 (UGC)

Nilson P., 1974, Catalogue of selected non-UGC galaxies, Uppsala Astr. Obs. Report 5

Paturel G., Fouqué P., Bottinelli L., Gouguenheim L., 1989,
A\&AS 80, 299

Paturel G., Petit C., Garnier R., Prugniel P., 1999, A\&AS 140, 89 (Paper II)

Paturel G., Fang Y., Petit C., Rousseau J., 2000, A\&A (submitted)

Rodgers A., Peterson B., Harding P., 1978, ApJ 225, 768

Roman A.T., Nakanishi K., Tomita A., Saito M., 1996, PASJ 48,679

Roman A.T., Nakanishi K., Saito M., 1998, PASJ 50, 37

Rood H.J., Baum W.A., 1967, AJ 72, 398

Saito M., et al., 1990, PASJ 42, 603

Saito M., et al., 1991, PASJ 43, 449

Takase B., Miyauchi-Isobe N., 1993, Publ. Natl. Astron. Obs. Jpn. 3, 169

Vorontsov-Velyaminov B.A., Arkipova V.P., Kranogorskaja A.A., 1962-1974, Proc. Sternberg State Astron. Inst. No. 32-33-34-38-46 (MCG)

Weinberger R., 1980, A\&AS 40, 123

Zwicky F., 1971, Catalogue of Selected Compact Galaxies and of Post-Eruptive galaxies

Zwicky F., Herzog E., Karpowicz M., Kowal C.T., 1961-1968, Catalogue of Galaxies and Cluster of Galaxies, California Institute of Tech., Pasadena, (Vols. I-VI) (CGCG)

Zwicky F., Sargent W.L.W., Kowal C., 1975, AJ 80, 545 